01

\title{
О распределении по размерам дисперсных частиц фрактальной формы
}

\author{
(C) В.Б. Федосеев, А.В. Шишулин
}

Институт металлорганической химии РАН им. Г.А. Разуваева, 603137 Нижний Новгород, Россия

e-mail: Chichouline_Alex@live.ru

Поступило в Редакцию 6 мая 2020 г.

В окончательной редакции 5 июля 2020 г.

Принято к публикации 16 июля 2020 г.

В рамках термодинамического подхода рассмотрена дисперсная система, образованная ансамблем частиц различных формы и объема. Форма частицы задана величиной ее фрактальной размерности, характеризующей связь объема и площади поверхности. С использованием методов теории чисел и формулы Харди-Рамануджана-Радемахера построены соответствующие состоянию термодинамического равновесия функции распределения по размерам частиц дисперсной фазы различной формы в ансамбле. На основе функций распределения получены оценки среднего размера и фрактальной размерности дисперсных частиц. Установлена взаимосвязь между средними геометрическими характеристиками частиц в ансамбле, термодинамическими условиями, в которых находится дисперсная система, и свойствами образующего ее вещества.

Ключевые слова: дисперсная система, наночастицы, распределение по размерам, фрактальная размерность, теория чисел.

DOI: $10.21883 /$ JTF.2021.01.50270.159-20

\section{Введение}

Уникальные свойства малых частиц и микро- и наноструктурных материалов на их основе, нетипичные для фаз макроскопического размера, в течение нескольких последних десятилетий привлекают значительный интерес исследователей [1]. Отличительной особенностью частиц малого объема является существенное возрастание влияния их морфологии на широкий спектр физических и физико-химических свойств (механических, теплофизических, магнитных, термоэлектрических и др.), которые для частиц различного объема и формы могут варьироваться в широких пределах. Например, уменьшение объема наночастицы сопровождается заметными изменениями набора параметров от механических [2,3] и магнитных характеристик [4,5] до температур фазовых переходов первого [6] и второго рода [7], термодинамических характеристик материала [8], реакционной способности [9] и т.д. Разнообразные методы синтеза наноразмерных частиц обсуждаются в [10].

Для микро- и наночастиц многокомпонентного состава подобные особенности проявляются в зависимости характеристических температур фазовых превращений (ликвидуса и солидуса $[6,11,12]$, верхней критической температуры растворения $[13,14])$, а также числа и равновесного состава сосуществующих фаз [6,11-14], объема частицы и геометрической конфигурации всех межфазных границ. Эти эффекты (наряду с рядом характерных для фазовых переходов в микро- и наноразмерных системах динамических особенностей [15]) зафиксированы экспериментально $[16,17]$ и могут быть смоделированы в рамках термодинамического [6,11-15] и молекулярно-динамического подходов [18]. Форма межфазных границ может быть охарактеризована с использованием различных безразмерных параметров (например, отношения площади поверхности фазы и площади поверхности сферы равного объема [13], а также ряда других $[11,14,19])$, включая фрактальную размерность [6,13]. С учетом данных особенностей ансамбли наночастиц различной морфологии не имеют фиксированных температур фазовых превращений и равновесных фазовых составов, которые оказываются „размытыми“ в некотором диапазоне [20]. Реализация размерных эффектов и эффектов формы при фазовых превращениях в наночастицах и их ансамблях может существенно изменить физико-химические свойства функциональных наноматериалов [13,21]. Таким образом, распределение по размерам частиц дисперсной фазы выступает одной из интегральных характеристик свойств дисперсной системы.

В настоящей работе рассматривается свободнодисперсная система (отсутствует взаимодействие между частицами дисперсной фазы), представленная ансамблем дисперсных частиц разного размера и формы. Форма частиц задается величиной их фрактальной размерности. Равновесные распределения частиц по размерам и форме находятся минимизацией свободной энергии дисперсной системы. Они позволяют оценить средний размер и фрактальную размерность частиц в зависимости от свойств вещества и внешних условий. Примерами оригинального применения полученных ниже закономерностей является развитая авторами настоящей 
работы методика диагностики состояния биологических объектов на основе анализа морфологии осадков, образующихся при кристаллизации веществ, растворенных в каплях биологических жидкостей [22], управляемое получение ансамблей дисперсных фаз с узкими би- и полимодальными распределениями по размерам [23], а также оценки влияния давления на геометрические особенности протяженных дефектов кристаллической структуры [24].

\section{1. Математическая модель ансамбля частиц свободнодисперсной системы}

Объектом моделирования является дисперсная система, представленная ансамблем частиц различного размера и заданной нерегулярной формы. В системе выполняется условие сохранения количества вещества в виде $N=\sum_{v} n_{v} v$, где $N-$ суммарное число атомов (молекул, мономеров и т.п.); v - стехиометрическое число частицы, равное количеству образующих ее атомов, которое далее будет отождествляться с размером частиц; $n_{v}$ - число частиц. Если условие сохранения вещества является единственным наложенным на дисперсную систему, то для описания характеристик ансамбля частиц возможно адаптировать подходы, основанные на теории разбиений [25]. В теории чисел разбиение натурального числа $N$ есть его представление в виде суммы натуральных чисел (где порядок чисел не учитывается), возможное число таких представлений называется числом разбиений $p(N)$. Согласно [26], каждое состояние дисперсной системы может быть представлено одним из разбиений.

Расчет числа разбиений $p(N)$ при заданном $N$ возможен с использованием, например, рекуррентной формулы Эйлера, диаграмм Юнга, графов Феррера или теории Харди-Рамануджана-Радемахера $[25,26]$, в рамках которой число разбиений представлено в виде сходящегося ряда. В случае больших $N$ для суммы данного ряда применима асимптотическая оценка [26]:

$$
p(N) \sim \exp \left(\pi \sqrt{\frac{2}{3}} \sqrt{N-\frac{1}{24}}\right) / 4 N \sqrt{3} .
$$

Таким образом, доля разбиений $f_{p}(N, v)$ числа $N$, содержащих слагаемое $v$, описывается выражением

$$
\begin{aligned}
f_{p}(N, v) & =\frac{N}{N-v} \exp \left\{\pi\left(\sqrt{\frac{2}{3}(N-v)}-\sqrt{\frac{2}{3} N}\right)\right\} \\
& \cong \exp \left(-\frac{\pi v}{\sqrt{6 N}}\right)
\end{aligned}
$$

полученным как отношение числа разбиений $p(N-v)$ к полному числу разбиений $p(N)$ (слагаемым $-1 / 24$ в (2) пренебрегли). Число разбиений $p(N)$ можно отождествить со статистической суммой, а функцию $f_{p}(N, v)$ рассматривать как вероятность существования в дисперсной системе частицы со стехиометрическим числом $v$.

В качестве равновесного распределения может быть использовано каноническое распределение Гиббса [27] $f_{v} \sim \exp \left(-U_{v} / R T\right)$, где $U_{v}-$ энергия моля вещества, разбитого на частицы размером $v, R$ - газовая постоянная, $T-$ температура. Энергию $U_{v}$ можно связать с поверхностной энергией или работой, затраченной на создание поверхности раздела при дроблении моля вещества на одинаковые частицы: $U_{v}=\sigma A_{v}, A_{v}=n_{v} a_{v}$. Здесь $\sigma$ - удельная поверхностная энергия на границе между дисперсной фазой и дисперсионной средой, $A_{v}$, $a_{v}$ - площади поверхности одного моля вещества и одной дисперсной частицы соответственно. Объем частицы $V_{v}$ однозначно связан с числом атомов в ней: $V_{v}=v V_{\text {mol }} / N_{\mathrm{A}}$, где $N_{\mathrm{A}}$ - число Авогадро, $V_{\text {mol }}-$ мольный объем. Площадь поверхности и линейные размеры частиц определяются как их объемом, так и формой (например, длина ребра куба равна $V_{v}^{1 / 3}$, диаметр сферы - $\left(6 V_{v} / \pi\right)^{1 / 3}$ и т.д.). Реальные частицы, как правило, обладают сложной нерегулярной формой, для описания которой удобно применение методов фрактальной геометрии $[6,13,21,22,24]$. В рамках данного подхода форма частиц задается величиной их фрактальной размерности, связывающей площадь поверхности частицы и ее стехиометрическое число: $A(v)=C v^{2 / D}$. Здесь $D-$ фрактальная размерность; множитель $C=k\left(V_{\text {mol }} / N_{\mathrm{A}}\right)^{2 / 3}$ согласует размерность и численно определяется мольным объемом вещества $V_{\text {mol }} ; N_{\mathrm{A}}$ - число Авогадро; $k$ - численный коэффициент; без ограничения общности дальнейших выводов положим $k=4 \pi$ [28]. По определению чем меньше $D$, тем сложнее форма дисперсных частиц, тем больше площадь поверхности и поверхностная энергия $U_{v}$ при равных $v$ (примеры фрактальных структур с различными $D$ приведены на рис. 1). Как отмечалось в [21], фрактальная геометрия, используя в качестве параметра соотношение площади поверхности и объема (ключевой параметр в термодинамике наноструктур), является одним из наиболее общих и удобных методов для описания формы максимально широкого класса геометрических объектов (от достаточно простых платоновых и архимедовых тел $[11,14,29]$, пластин, многогранников нерегулярной формы [30-32] до нанотрубок, вискеров, дендритоподобных объектов [33] и структур, подобных изображенным на рис. 1).

Полагая число разбиений и энергию частиц независимыми величинами (при расчете свободной энергии образования рассматриваются только индивидуальные характеристики частицы, которые для невзаимодействующих частиц (свободнодисперсная система) не зависят от характеристик других частиц, таким образом, распределение частиц по энергиям также не зависит от реализуемого разбиения), единое распределение получим перемножением функций $f_{v}$ и $f_{p}$, что эквивалентно 

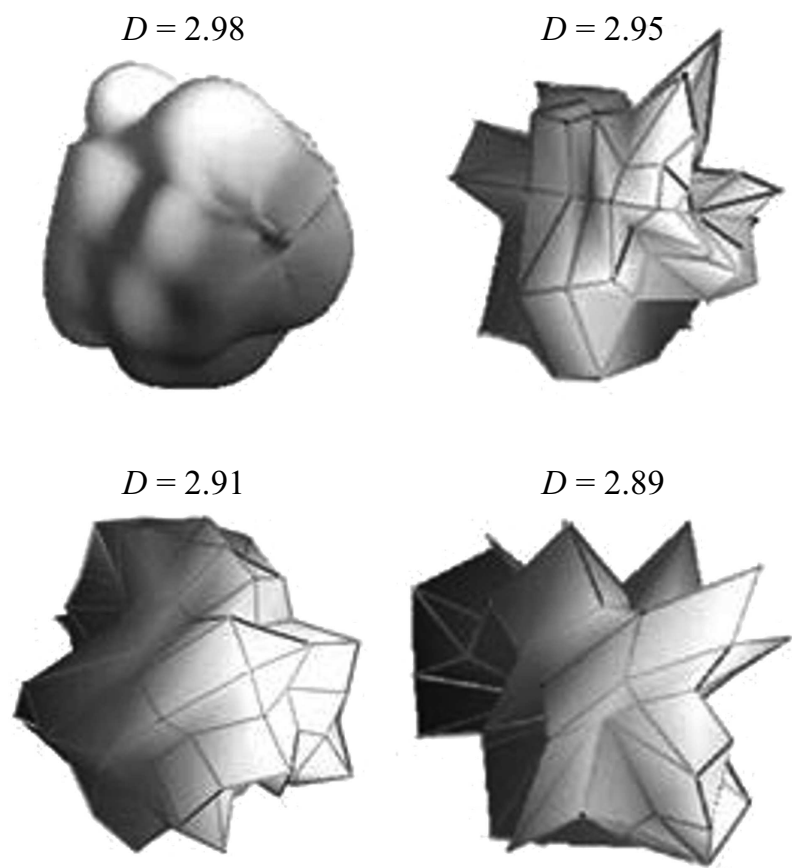

Рис. 1. Примеры фрактальных структур с различными $D$.

добавлению слагаемого $R T \ln f_{p}$ к энергии $U_{v}$

$$
f_{D}(v, D, N) \sim f_{p} f_{v} \sim \exp \left(-\frac{U_{v}+R T \ln f_{p}}{R T}\right) .
$$

Величина $G(v, D, N)=U_{v}+R T \ln f_{p} \quad$ соответствует свободной энергии дисперсной системы (на моль вещества), а $R T \ln f_{p}$ - энтропийному вкладу, связанному с диспергированием системы.

Оценки области применимости термодинамического подхода при описании структур малого объема на основе теории флуктуаций получены в [34]. Подобные результаты могут быть дополнены рассмотрением размерных зависимостей поверхностной энергии наночастиц [35], а также ряда физических и физико-химических свойств, включая обусловленные квантовыми эффектами [36]. В соответствии с данными [34-36] термодинамический подход успешно применяется для прогнозирования равновесных состояний в ансамблях нанодисперсных частиц и фазовых превращений в структурах малого объема при характерных размерах частиц вплоть до 2-5 nm (см., например, результаты $[37,38]$ ).

\section{2. Результаты и их обсуждение}

Вид распределения $f_{p}$ и распределений $f_{D}$ с разными значениями фрактальной размерности приведен на рис. 2. Следует отметить, что одно и то же значение фрактальной размерности $D$ соответствует набору частиц различной геометрической конфигурации, объединенным одним и тем же соотношением количества вещества и площади поверхности (см., например, рис. 1, $b$ в [21]). Также, например, конические, пирамидальные, эллипсоидальные и другие частицы с одинаковым $D$ равной степени, соответствующие $D=3.00$, могут одновременно присутствовать в дисперсной системе, не искажая распределения (3).

Во врезке на рис. 2, а приведен характерный вид гистограммы, полученной из распределений $f_{D}$, иллюстрирующей зависимость от $v$ количества $n_{D}(v)$ наночастиц с фрактальной размерностью $D$ и стехиометрическим числом $v$ в ансамбле. Приведенная зависимость получена из интегрального выражения, $n_{D}(v)=\int_{N_{0}}^{N_{0}+\Delta N} f_{D}(v, D, N) d v$, где $\left[N_{0}, N_{0}+\Delta N\right]$ - диапазон рассматриваемых стехиометрических чисел, и соответствует экспериментально полученным гистограммам для наночастиц [9,39-42], а также трехмерных элементов кристаллической структуры $[43,44]$. Интересным также является то, что подобный вид имеют и гистограммы распределений по размерам пор в поликристалле [45].
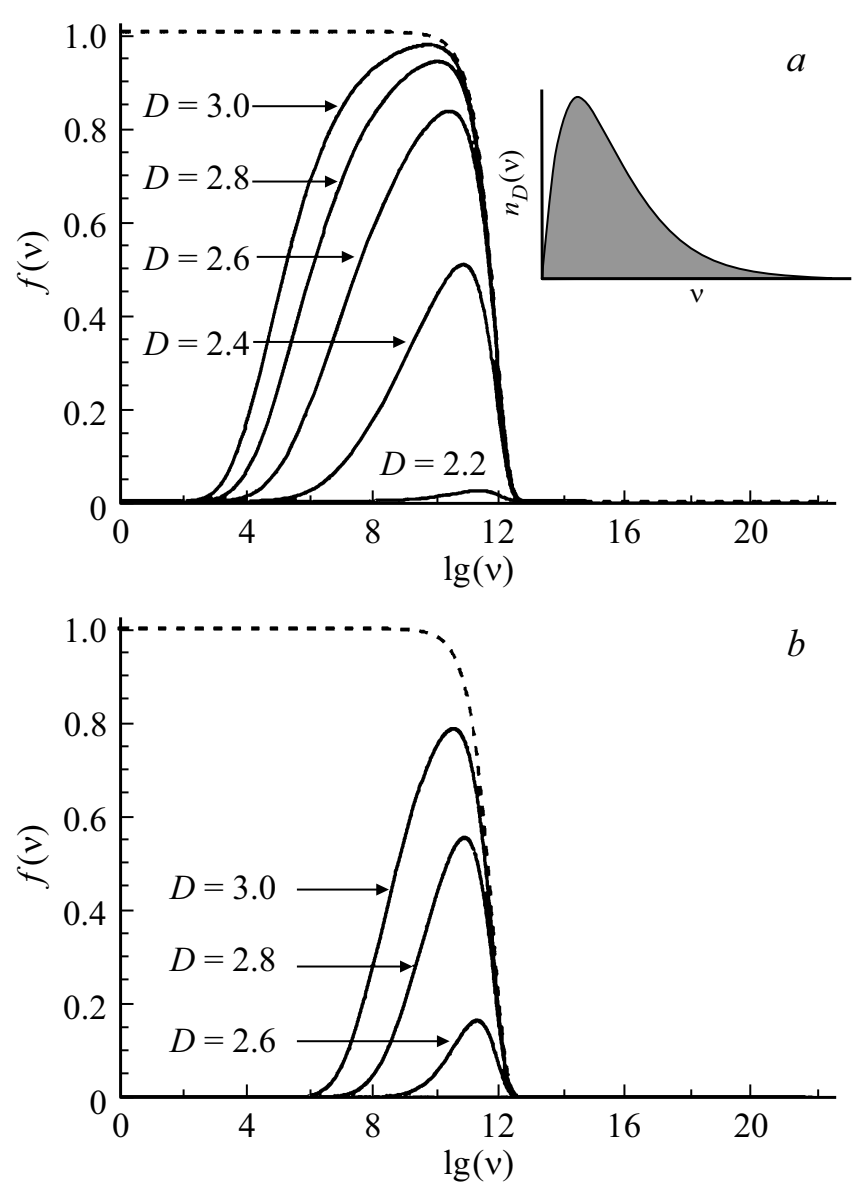

Рис. 2. Штрихом показана функция $f_{p}$, сплошные кривые соответствуют распределению $f_{D}$ для частиц с разной фрактальной размерностью, от верхней к нижней $D$ меняется с шагом 0.2 для: $a-$,льда“ $\left(\sigma=0.1 \mathrm{~J} / \mathrm{m}^{2}, V_{m o l}=18 \mathrm{~cm}^{3} / \mathrm{mol}\right.$, $T=273 \mathrm{~K}), b-$ „вольфрама“" $\left(\sigma=3 \mathrm{~J} / \mathrm{m}^{2}, V_{\text {mol }}=10 \mathrm{~cm}^{3} / \mathrm{mol}\right.$, $T=298 \mathrm{~K})$. На врезке показан характерный вид гистограммы для распределений $f_{D}$. 

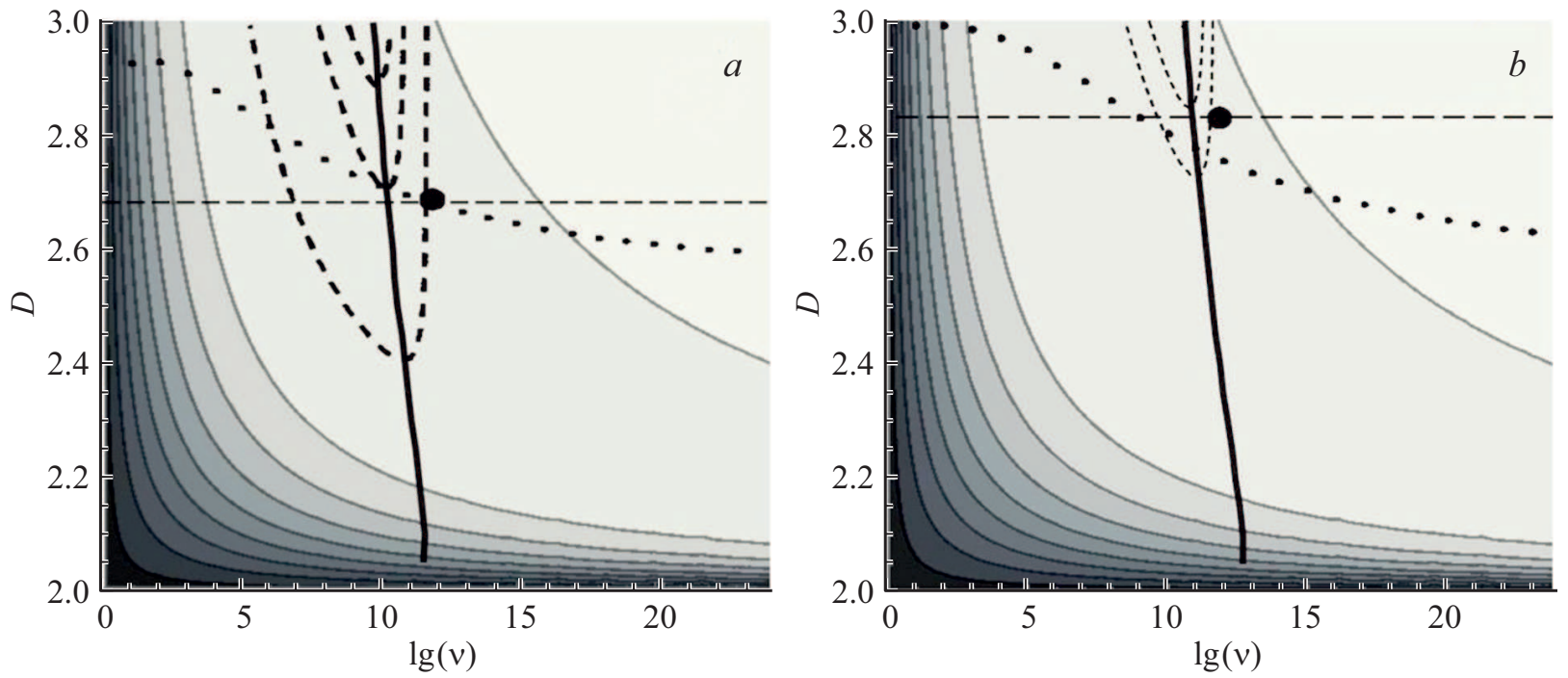

Рис. 3. Зависимость мольной поверхностной энергии монодисперсной системы $U_{v}$ от размера и формы частиц. Линия отображает положение минимумов свободной энергии $G(v, D, N)$ при вариации $D$, точкой обозначено состояние с координатами $(\langle v\rangle,\langle D\rangle)$ (дополнительно выделено горизонтальным пунктиром), пунктирными кривыми обозначены изолинии свободной энергии. Величина $\langle D\rangle$ получена на основе выборки распределений с шагом 0.05 . Насыщенность окраски возрастает с ростом $U_{v}$. При построении использованы следующие характеристики веществ: $a-$ „лед“ $\left(\sigma=0.1 \mathrm{~J} / \mathrm{m}^{2}, V_{m o l}=18 \mathrm{~cm}^{3} / \mathrm{mol}\right), b-$ „вольфрам“ $\left(\sigma=3 \mathrm{~J} / \mathrm{m}^{2}, V_{m o l}=10 \mathrm{~cm}^{3} / \mathrm{mol}\right)$.

Функция позволяет оценить некоторые свойства дисперсной системы. Максимум распределения по размерам соответствует минимуму $G(v, D, N)$. Средние величины фрактальной размерности и стехиометрического числа частиц $\langle v\rangle$ могут быть оценены на основе выборки распределений для $D_{i} \in(2,3]$ с произвольно выбранным шагом, как

$$
\langle D\rangle=\frac{\sum_{i} D_{i} f_{D}\left(D_{i}, v, N\right)}{\sum_{i} f_{D}\left(D_{i}, v, N\right)},\langle v\rangle=\frac{\sum_{i} v f_{D}\left(D_{i}, v, N\right)}{\sum_{i} f_{D}\left(D_{i}, v, N\right)} .
$$

Аналогично можно описать другие свойства дисперсной системы, например, оценить эффективный линейный размер частиц как длину ребра куба равного объема $\langle d\rangle=d_{1} \sum_{i} v^{1 / 3} f_{D}\left(D_{i}, v, N\right) / \sum_{i} f_{D}\left(D_{i}, v, N\right)$ либо средний диаметр фрактальной частицы $\left\langle d^{*}\right\rangle=$ $=d_{1} \sum_{i} v^{1 / D} f_{D}\left(D_{i}, v, N\right) / \sum_{i} f_{D}\left(D_{i}, v, N\right)$, где $d_{1}$ - линейный размер атома. Очевидно, что линейный размер фрактальной частицы больше, чем у куба эквивалентного объема $\left\langle d^{*}\right\rangle>\langle d\rangle$. Отметим, что среднее стехиометрическое число частицы $\langle v\rangle$ и средний диаметр $\langle d\rangle$ не связаны соотношением $\langle d\rangle \sim\langle v\rangle^{1 / 3}$. Необходимо принять во внимание, что некоторого уточнения полученных результатов можно достичь путем дополнительного учета энтропийного вклада, соответствующего наличию множества состояний с равным $D$. В аналитическом виде данный вклад может быть получен, например, с использованием энтропии различий Кульбака-Лейблеpa [46], и следует ожидать в этом случае точной локализации минимума свободной энергии дисперсной системы около состояния с $D \approx\langle D\rangle$ и $v \approx\langle v\rangle$. Также большая детализация полученных результатов может быть достигнута учетом того, что переходной слой от объемного материала ядра дискретной частицы к ее поверхности может включать несколько подповерхностных зон с постепенным уменьшением их заполнения веществом в интервале размерностей от $D \in(2,3)$ вплоть до субмонослойной нестехиометрической зоны на границе контакта дисперсной фазы и дисперсионной среды с размерностью $D \in(1,2)$ и даже с $D \in(0,1)$; для уточненной характеризации фрактальной формы частицы требуется применение спектра мультифрактальных размерностей.

Зависимость поверхностной энергии монодисперсной системы, содержащей один моль вещества, $U_{v}$, от размера $v$ и фрактальной размерности $D$ частиц приведена на рис. 3 в виде поверхностей свободной энергии в координатах „логарифм стехиометрического числа частицы - ее фрактальная размерность“. На нем показаны расположение в данных координатах изолиний свободной энергии $G(v, D, N)$, а также изменение положения ее минимумов в зависимости от фрактальной размерности $D$ частиц в ансамбле. На рис. 3 величина мольной поверхностной энергии отражена насыщенностью окраски, возрастающей с ростом $U_{v}$ и соответствующей возрастанию поверхностной энергии при диспергировании и усложнении формы частиц дисперсной фазы. Состояние в верхнем правом углу диаграммы в точке $\left(\lg \left(N_{\mathrm{A}}\right), 3\right)$ соответствует единственной частице, имеющей регулярную форму (сфера, куб), состояния слева (в точке $(\lg (v)=0))$ соответствуют полной атомизации 
системы, при которой фрактальная размерность теряет физический смысл.

Основным результатом предложенного описания является утверждение о том, что в общем случае нерегулярная форма частиц является наиболее характерной для дисперсных систем. Это проявляется при формировании частиц путем диспергирования и ансамблей зерен поликристаллического материала и может быть проиллюстрировано снимками, приведенными, например, в $[9,17,22,39,40,42,47]$. Отметим, что данная особенность подтверждается имеющимися результатами $[1,48,49]$, свидетельствующими о нестабильности формы частиц малого объема, обладающих четкой огранкой.

Уравнения (3)-(6) служат иллюстрацией того, что в находящейся в термодинамическом равновесии дисперсной системе средний размер и морфология частиц зависят от количества вещества, его свойств, а также дисперсионной среды, задающей величину $\sigma$. При этом увеличение количества вещества, составляющего дисперсную систему, сопровождается нелинейным ростом эффективного размера частиц с одновременным уменьшением их фрактальной размерности. В свою очередь, с ростом $\sigma$ или $V_{\text {mol }}$ максимум распределения по размерам (5) смещается в сторону крупных частиц, а средняя фрактальная размерность (6) возрастает. При понижении температуры, согласно (5), следует ожидать того же эффекта. Экспериментально роль условий формирования дисперсной системы демонстрирует пример образования прозрачных и непрозрачных пленок хитозана с различной фрактальной структурой кристаллических включений бензойной кислоты из одного исходного состояния, приведенный в работе [50].

Другим следствием повышения поверхностной энергии либо понижения температуры является формирование более узких распределений по размерам, примеры подобных распределений рассматривались в [23]. Некоторые замечания о пределе сужения распределения по размерам частиц дисперсной фазы приведены в [51].

Оценка фрактальной размерности частиц по данным оптической и электронной микроскопии может осуществляться с высокой точностью в рамках целого ряда подходов [52]. Ранее нами был предложен один из таких подходов, представляющий собой модифицированный ретикулярный метод подсчета клеток [53] (box-counting technique в англоязычных источниках). Для двумерного случая практическая реализация данного подхода описана в [50] и осуществлена авторами для исследования структур высыхающих капель биологических растворов как одного из средств диагностики состояния живых организмов [22].

\section{Заключение и выводы}

1. На основе методов равновесной термодинамики и теории разбиений построена модель ансамбля частиц свободнодисперсной системы и получены функции распределения по размерам частиц различной формы в ансамбле. В рамках предложенного подхода форма частицы задается величиной ее фрактальной размерности $D$, при этом каждому ее значению соответствует множество эквивалентных между собой геометрических конфигураций, которые могут одновременно присутствовать в системе, не изменяя функций распределения.

2. Средние значения геометрических характеристик частиц ансамбля определяются комплексом факторов, включающих как характеристики вещества (мольные объемы, поверхностные энергии на границе раздела с дисперсионной средой), так и количество вещества, образующего дисперсную фазу, и термодинамические условия (температуру). При этом предложенная модель при, например, уменьшении количества вещества в системе позволяет ожидать уменьшения средних значений объема частицы и фрактальной размерности (,ууложнения“ формы частицы), а, например, большим величинам мольных объемов и поверхностных натяжений соответствуют большие величины средних объема и $D$. В общем случае нерегулярная форма частиц дисперсной фазы $(D<3.00)$ является наиболее характерной для дисперсных систем.

\section{Благодарности}

Авторы благодарят двух рецензентов за внимание к работе и ценные замечания.

\section{Финансирование работы}

Работа выполнена в соответствии с государственным заданием ИМХ РАН, а также при поддержке Российского фонда фундаментальных исследований (проект 18-08-01356-a).

\section{Конфликт интересов}

Авторы заявляют, что у них нет конфликта интересов.

\section{Список литературы}

[1] С.А. Непийко. Физические свойства малых металлических частии, (Наук. думка, Киев, 1985).

[2] М.Н. Магомедов. ЖТФ, 85 (6), 152 (2015) [M.N. Magomedov. Tech. Phys., 60 (6), 937 (2015). DOI: $10.1134 / \mathrm{S} 106378421506016 \mathrm{X}$

[3] М.Н. Магомедов. ФТТ, $\mathbf{6 1}(1), \quad 148 \quad$ (2019). DOI: $10.21883 /$ FTT.2019.01.46905.175

[M.N. Magomedov. Phys. Solid State, 61 (1), 23 (2019) DOI: 10.1134/S1063783419010165]

[4] В.Н. Никифоров, А.Н. Игнатенко, В.Ю. Ирхин. ЖЭТФ, 151 (2), 356 (2017) [V.N. Nikiforov, A.N. Ignatenko, V.Yu. Irkhin. J. Exp. Theor. Phys., $124(2), 304$ (2017). DOI: $10.1134 /$ S1063776117010046] 
[5] С.В. Столяр, С.В. Комогорцев, Л.А. Чеканова, Р.Н. Ярославцев, О.А. Баюков, Д.А. Великанов, М.Н. Волочаев, Е.В. Черемискина, M.Sh. Bairmani, П.Е. Ерошенко, Р.С. Исхаков. Письма в ЖТФ, 45 (17), 28 (2019). DOI: 10.21883/PJTF.2019.17.48220.17886

[S.V. Stolyar, S.V. Komogortsev, L.A. Chekanova, R.N. Yaroslavtsev, O.A. Bayukov, D.A. Velikanov, M.N. Volochaev, E.V. Cheremiskina, M.Sh. Bairmani, P.E. Eroshenko, R.S. Iskhakov. Tech. Phys. Lett., 45(9), 878 (2019) DOI: $10.1134 / \mathrm{S} 1063785019090116]$

[6] А.В. Шишулин, В.Б. Федосеев, А.В. Шишулина. ЖТФ, 89 (9), 1420 (2019). DOI: 10.21883/JTF.2019.09.48069.88-19 [A.V. Shishulin, V.B. Fedoseev, A.V. Shishulina. Tech. Phys., 64 (9), 1343 (2019). DOI: 10.1134/S1063784219090172]

[7] C.C. Yang, Q. Jiang. Acta Mater., 53 (11), 3305 (2005). DOI: $10.1016 /$ j.actamat.2005.03.039

[8] М.H. Магомедов. ФТТ, 61 (4), $757 \quad$ (2019). DOI: $10.21883 /$ FTT.2019.04.47426.267

[M.N. Magomedov. Phys. Solid State, 61 (4), 642 (2019). DOI: $10.1134 / \mathrm{S} 106378341904019 \mathrm{X}]$

[9] А.В. Калинкин, А.М. Сорокин, М.Ю. Смирнов, В.И. Бухтияров. Кинет. катал., 55 (3), 371 (2014). [A.V. Kalinkin, A.M. Sorokin, M.Y. Smirnov, V.I. Bukhtiyarov. Kinet. Catal., 55 (3), 354 (2014) DOI: 10.1134/S0023158414030045]

[10] М.К. Бернер, В.Е. Зарко, М.Б. Талавар. Физика горения и взрыва, 49 (6), 3 (2013 [M.K. Berner, V.E. Zarko, M.B. Talawar. Combust. Explos. Shock Waves, $49(6), 625$ (2013). DOI: 10.1134/S0010508213060014]

[11] R. Mendoza-Pérez, G. Guisbiers. Nanotechnology, 30, 305702 (2019). DOI: 10.1088/1361-6528/ab1759

[12] A. Shirinyan, G. Wilde, Yu. Bilogorodskyy. J. Mater. Sci., 55, 12385 (2020). DOI: 10.1007/s10853-020-04812-2

[13] А.В. Шишулин, В.Б. Федосеев, А.В. Шишулина. ЖТФ, 89 (4), 556 (2019). DOI: 10.21883/JTF.2019.04.47311.343-18 [A.V. Shishulin, V.B. Fedoseev, A.V. Shishulina. Tech. Phys., 64 (4), 512 (2019). DOI: 10.1134/S1063784219040200]

[14] L.-D. Geoffrion, G. Guisbiers. J. Phys. Chem. C, $124(25)$, 14061 (2020). DOI: 10.1021/acs.jpcc.0c04356

[15] Е.Н. Федосеева, В.Б. Федосеев. ЖТФ, 90 (6), 879 (2020). DOI: $10.21883 /$ JTF.2020.06.49270.23-19

[16] B. Straumal, B. Baretzky, A. Mazilkin, S. Protasova, A. Myatiev, P. Straumal. J. Eur. Ceram. Soc., 29 (10), 1963 (2009). DOI: 10.1016/j.jeurceramsoc.2009.01.005

[17] G. Radnóczi, E. Bokányi, Z. Erdélyi, F. Misják. Acta Mater., 123, 82 (2017). DOI: 10.1016/j.actamat.2016.10.036

[18] Y. Magnin, A. Zappelli, H. Amara, F. Ducastelle, C. Bichara. Phys. Rev. Lett., 115, 205502 (2015). DOI: $10.1103 /$ physrevlett.115.205502

[19] М.Н. Магомедов. ЖТФ, 86 (5), 84 (2016.) [M.N. Magomedov. Tech. Phys., 61 (5), 722 (2016). DOI: $10.1134 / \mathrm{S} 1063784216050145]$

[20] М.Н. Магомедов. В сб. Физико-химические аспекты изучения кластеров. наноструктур, наноматериалов, под ред. В.М. Самсонова, Н.Ю. Сдобнякова. (Твер. гос. ун-т, Тверь, 2013), с. 169.

[21] А.В. Шишулин, В.Б. Федосеев. Кинет. катал., 60 (3), 334 (2019). [A.V. Shishulin, V.B. Fedoseev. Kinet. Catal., 60 (3), 315 (2019). DOI: 10.1134/S0023158419030121]

[22] О.А. Голованова, Е.С. Чиканова, В.Б. Федосеев. Кристаллогр., 63 (3), 471 (2018). [O.A. Golovanova, E.S. Chikanova, V.B. Fedoseev. Crystallogr. Rep., 63(3), 493 (2018). DOI: $10.7868 / \mathrm{S} 0023476118030190]$
[23] В.Б. Федосеев, Е.Н. Федосеева. ИФЖ, 92 (5), 2229 (2019). [V.B. Fedoseev, E.N. Fedoseeva. J. Eng. Phys. Thermophys., 92 (5), 1191 (2019). DOI: 10.1007/s10891-019-02033-2]

[24] В.Б. Федосеев. Бутлеров. сообщ., 23 (14), 36 (2010).

[25] Г. Эндрюс. Теория разбиений. (Наука, М., 1982)

[26] G.H. Hardy, S. Ramanujan. Proc. Lond. math. soc., 17, 75 (1918).

[27] Л.Д. Ландау, Е.М. Лифшиц. Статистическая физика (Теоретическая физика, m. V). (Физматлит, М. 2005)

[28] A.V. Shishulin, V.B. Fedoseev. J. Mol. Liq., 278, 363 (2019). DOI: 10.1016/j.molliq.2019.01.050

[29] Cui M., H. Lu, H. Jiang, Z. Cao, X. Meng. Sci. Rep. 7, 41990 (2017). DOI: $10.1038 /$ srep41990

[30] B.R. Cuenya. Thin Solid Films, 518(12), 2127 (2010). DOI: 10.1016/j.tsf.2010.01.018

[31] J.-P. Polomares-Báez, J.-M. Montejano-Carrizalez, G. Guisbiers, M. José-Yacamán, J.-L. Rodríguez-López. Nanotechnology, 30, 425791 (2019). DOI: 10.1088/1361-6528/ab27eb

[32] J.J. Velazquez-Salazar, L. Bazán-Díaz, Q. Zhang, R. Mendoza-Cruz, L. Montaño-Priede, G. Guisbiers, N. Large, S. Link, M. José-Yacamán. ACS Nano, 13 (9), 10113 (2019). DOI: 10.1021/acsnano.9b03084

[33] F.-X. Niu, Y.-X. Wang, L.-R. Ma, S.-L. Fu, I. Abbas, C. Qu, C.-G. Wang. J. Alloys Cmpd., 714, 270 (2017). DOI: $10.1016 /$ j.jallcom.2017.04.186

[34] В.М. Самсонов, Д.Э. Деменков, В.И. Карачаров, А.Г. Бембель. Изв. РАН. Сер. физ., 75 (8), 1133 (2011). [V.M. Samsonov, D.E. Demenkov, V.I. Karacharov, A.G. Bembel. Bulletin of the Russian Academy of Sciences: Physics, 75 (8), 1073 (2011). DOI: 10.3103/S106287381108034X]

[35] В.М. Самсонов, А.А. Чернышова, Н.Ю. Сдобняков. Изв. PAH. Сер. физ., 80 (6) 768 (2016). [V.M. Samsonov, A.A. Chernyshova, N.Yu. Sdobnyakov. Bulletin of the Russian Academy of Sciences: Physics, 80 (6), 698 (2016). DOI: $10.3103 /$ S1062873816060290]

[36] M. Wautelet, D. Duvivier. Eur. J. Phys., 28, 953 (2007). DOI: $10.1088 / 0143-0807 / 28 / 5 / 018$

[37] G. Kaptay. J. Mater. Sci., 47, 8320 (2012). DOI: $10.1007 / \mathrm{s} 10853-012-6772-9$

[38] M. Monji, M.A. Jabbareh. CALPHAD, 58, 1 (2017). DOI: 10.1016/j.calphad.2017.04.003

[39] X. He, W. Zhong, C.-T. Au, Y. Du. Nanoscale Res. Lett., 8, 446 (2013). DOI: 10.1186/1556- 276X-8-446

[40] М.Ю. Смирнов, А.В. Калинкин, Е.И. Вовк, В.И. Бухтияров. Кинет. катал., 56 (6), 791 (2015). [M.Y. Smirnov, A.V. Kalinkin, E.I. Vovk, V.I. Bukhtiyarov. Kinet. Catal., 56 (6), 801 (2015). DOI: 10.1134/S0023158415060129]

[41] В.В. Карасев, А.А. Онищук, С.А. Хромова, О.Г. Глотов, В.Е. Зарко, Е.А. Пилюгина, Ч.-Тз. Цзай. Физика горения и взрыва, 42 (6), 33 (2006). [V.V. Karasev, A.A. Onishchuk, S.A. Khromova, O.G. Glotov, V.E. Zarko, E.A. Pilyugina, C.J. Tsai. Combust. Explos. Shock Waves, 42 (6), 649 (2006). DOI: 10.1007/s10573-006-0098-3]

[42] M. Khakbiz, F. Akhlaghi, P.S. Bagha, L. Ghazanfari. Phys. B, 556, 132 (2019). DOI: 10.1016/j.physb.2018.12.033

[43] M.V. Degtyarev, T.I. Chashchukhina, L.M. Voronova, A.M. Patselov, V.P. Pilyugin. Acta Mater., 55, 6039 (2007). DOI: $10.1016 /$ j.actamat.2007.04.017 
[44] Т.И. Чащухина, Л.М. Воронова, М.В. Дегтярев. Изв. PAН. Сер. физ., 71 (2), 283 (2007). [T.I. Chashchukhina, L.M. Voronova, M.V. Degtyarev. Bulletin of the Russian Academy of Sciences: Physics, $71(2), 275$ (2007). DOI: $10.3103 / \mathrm{S} 1062873807020335]$

[45] V.N. Chuvil'deev, A.V. Nokhrin, V.I. Kopylov, M.S. Boldin, M.M. Vostokov, M.Yu. Gryaznov, N.Yu. Tabachkova, P. Tryaev. J. Mater. Sci., $54(24) .14926$ (2019). DOI: $10.1007 / \mathrm{s} 10853-019-03926-6$

[46] А.М. Агаларов, А.А. Потапов, А.Э. Рассадин, А.В. Степанов. Моделирование и анализ информационных систем, 25 (1), 7 (2018). DOI: 10.18255/1818-1015-2018-1-7-17

[47] П.П. Федоров, В.В. Осико. ДАН, 488 (3), 253 (2019). [P.P. Fedorov, V.V. Osiko. Doklady Physics, $64(9), 353$ (2019). DOI: 10.1134/S1028335819090076]

[48] Э.Л. Нагаев. УФН, 162 (9), 49 (1992).

[49] Е.Б. Долгушева, В.Ю. Трубицын. ФТТ, 52 (6), 1163 (2010) [E.B. Dolgusheva, V.Y. Trubitsin. Phys. Solid State, 52 (6), 1238 (2010). DOI: 10.1134/S1063783410060193]

[50] Е.Н. Федосеева, В.Б. Федосеев. Высокомол. соед. Сер. А, 53 (11), 1900 (2011). [E.N. Fedoseeva, V.B. Fedoseev. Polymer science. Ser. A, 53(11), 1040 (2011). DOI: 10.1134/S0965545X1110004X]

[51] V. Burlakov, A. Goriely. Europhys. Lett., 119(5), 50001 (2017). DOI: 10.1209/0295-5075/119/50001

[52] О.И. Шелухин, Д.И. Магомедова. Наукоемкие технологии в космических исследованиях Земли, 9 (6), 6 (2017).

[53] J. Li, Q. Du, C. Sun. Pattern recognition, 42, 2460 (2009). DOI: 10.1016/j.patcog.2009.03.001 